\title{
Long-time behavior of a semilinear wave equation with memory
}

\author{
Baowei Feng ${ }^{1}$, Maurício L Pelicer ${ }^{2 *}$ and Doherty Andrade ${ }^{3}$
}

"Correspondence: peliceri@gmail.com

${ }^{2}$ Department of Sciences, Regional Campus of Goioerê, State University of Maringá, Goioerê, Paraná 87360-000, Brazil

Full list of author information is available at the end of the article

\section{Abstract}

In this paper we study the long-time dynamics of the semilinear viscoelastic equation

$$
u_{t t}-\Delta u_{t t}-\Delta u+\int_{0}^{\infty} \mu(s) \Delta u(t-s) d s+f(u)=h
$$

defined in a bounded domain of $\mathbb{R}^{3}$ with Dirichlet boundary condition. The functions $f=f(u)$ and $h=h(x)$ represent forcing terms and the kernel function $\mu \geq 0$ is assumed to decay exponentially. Then, by exploring only the dissipation given by the memory term, we establish the existence of a global attractor to the corresponding dynamical system.

MSC: 35L71; 35B41;74D99

Keywords: wave equation; global attractor; memory; viscoelasticity

\section{Introduction}

This is paper is concerned with the long-time behavior of a class of wave equations with memory of the form

$$
\begin{aligned}
& u_{t t}-\Delta u_{t t}-\Delta u+\int_{0}^{\infty} \mu(s) \Delta u(t-s) d s+f(u)=h \quad \text { in } \Omega \times \mathbb{R}^{+}, \\
& u=0 \quad \text { on } \Gamma \times \mathbb{R}^{+},
\end{aligned}
$$

with initial conditions

$$
\begin{aligned}
& u(x, 0)=u_{0}(x), \quad u_{t}(x, 0)=v_{0}(x), \quad \forall x \in \Omega, \\
& u(x,-s)=\varphi(x, s), \quad \forall(x, s) \in \Omega \times \mathbb{R}^{+},
\end{aligned}
$$

where $\Omega$ is a bounded domain in $\mathbb{R}^{3}$ with smooth boundary $\Gamma$, and $\varphi$ is a prescribed past history.

This problem is related to a model of extensional vibrations of thin rods

$$
u_{t t}-\Delta u_{t t}-\Delta u=0
$$

described in Love [1], Chapter 20, which is a conservative system. Here, we have added a nonlinear forcing $f(u)$ and a dissipation of memory type.

(c) 2016 Feng et al. This article is distributed under the terms of the Creative Commons Attribution 4.0 International License (http://creativecommons.org/licenses/by/4.0/), which permits unrestricted use, distribution, and reproduction in any medium, provided you give appropriate credit to the original author(s) and the source, provide a link to the Creative Commons license, and indicate if changes were made. 
We observe that such a system was extensively studied in the more general form

$$
\left|u_{t}\right|^{\rho} u_{t t}-\Delta u_{t t}-\Delta u+\int_{0}^{\infty} \mu(s) \Delta u(t-s) d s+f(u)=0
$$

with $0 \leq \rho<2$. Most of results are concerned with the exponential stability of the system under additional damping $-\Delta u_{t}$ or $u_{t}$. We refer the reader to, e.g., [2-5]. The existence of global attractors to (1.4) was first proved in Araújo et al. [6], with the assumption $\rho>1$ and with the additional damping $-\Delta u_{t}$. The assumption $\rho>1$ was technical and related to the uniqueness of the problem. Later, it was shown in [7] that the strong damping $-\Delta u_{t}$ could be replaced by the weak damping $u_{t}$, but yet with $\rho>1$. On the other hand, in [8], the existence of a global attractor for the problem with $\rho=\mu=0$ was studied with a strong damping.

More recently, it was proved by Conti et al. [9] that existence and uniqueness for the mixed problem (1.4) holds for $\rho \geq 0$ and without additional damping terms, that is, keeping only the dissipation given by the memory. This means that the restriction $\rho>1$ can be dropped.

Motivated by results in [6] and [9], we propose to study the existence of global attractors of (1.4) with $\rho=0$ and exploring only the dissipation given by the memory term. That is, we consider the problem (1.1)-(1.3). Then our result extends or complements the ones in [6-9]. See Theorem 3.1.

Of course, if the rotational inertia $\Delta u_{t t}$ is dropped, then equation (1.1) becomes the wellknown viscoelastic wave equation of memory type. On this matter, we refer the reader to some relevant results in [10-14], among others.

\section{History setting}

We denote by $(\cdot, \cdot)$ and $\|\cdot\|$ is the standard inner product and norm on $L^{2}(\Omega)$. It is well known that the operator $A$ with domain $D(A)$ defined by

$$
A=-\Delta, \quad D(A)=H^{2}(\Omega) \cap H_{0}^{1}(\Omega),
$$

is self-adjoint and strictly positive. See, e.g., [15]. We adopt the notation

$$
H_{0}=L^{2}(\Omega), \quad H_{1}=H_{0}^{1}(\Omega) \quad \text { and } \quad H_{2}=H^{2}(\Omega) \cap H_{0}^{1}(\Omega) .
$$

Next we establish the history setting of the problem (1.1)-(1.3) in order to deal with the non-autonomous character of the memory term in (1.1). We follow the arguments of $[9$, $12,14]$, based on [16]. Let $\mu: \mathbb{R}^{+} \rightarrow[0, \infty)$ be a summable function. We denote by $\mathcal{M}$ the $L^{2}$-weighted space defined by

$$
L_{\mu}^{2}\left(\mathbb{R}^{+} ; H_{1}\right)=\left\{\eta: \mathbb{R}^{+} \rightarrow H_{1}:\|\eta\|_{\mathcal{M}}<\infty\right\}
$$

where $\|\cdot\|_{\mathcal{M}}=(\cdot, \cdot)_{\mathcal{M}}^{\frac{1}{2}}$ and $(\eta, \xi)_{\mathcal{M}}=\int_{0}^{\infty} \mu(\tau)(\eta(\tau), \xi(\tau))_{1} d \tau$. Similarly we define the space $\mathcal{M}_{0}$ as

$$
L_{\mu}^{2}\left(\mathbb{R}^{+} ; H_{0}\right)=\left\{\eta: \mathbb{R}^{+} \rightarrow H_{0}:\|\eta\|_{\mathcal{M}_{0}}<\infty\right\}
$$


where $\|\cdot\|_{\mathcal{M}_{0}}=(\cdot, \cdot)_{\mathcal{M}_{0}}^{\frac{1}{2}}$ and $(\eta, \xi)_{\mathcal{M}_{0}}=\int_{0}^{\infty} \mu(\tau)(\eta(\tau), \xi(\tau))_{0} d \tau$. From classical theory, the spaces $\mathcal{M}$ and $\mathcal{M}_{0}$ are separable Hilbert spaces.

Let $T$ be the infinitesimal generator of the right-translation semigroup on $\mathcal{M}$, that is,

$$
T \eta=-\eta^{\prime}
$$

for all $\eta \in D(T)=\left\{\eta \in \mathcal{M}: \eta^{\prime} \in \mathcal{M}, \eta(0)=0\right\}$, where $\eta^{\prime}(t)=\frac{\partial \eta}{\partial t}$ in the sense of distributions and $\eta(0)=\lim _{s \rightarrow 0} \eta(s)$. It is well known that

$$
(T \eta, \eta)_{\mathcal{M}} \leq 0, \quad \forall \eta \in D(T) .
$$

We also introduce the Hilbert space

$$
\mathcal{H}=H_{1} \times H_{1} \times \mathcal{M}
$$

endowed with the norms $\|\cdot\|_{\mathcal{H}}=(\cdot, \cdot)_{\mathcal{H}}^{\frac{1}{2}}$ where

$$
\left(\left(u_{1}, v_{1}, \eta_{1}\right),\left(u_{2}, v_{2}, \eta_{2}\right)\right)_{\mathcal{H}}=\left(u_{1}, u_{2}\right)_{1}+\left(v_{1}, v_{2}\right)_{1}+\left(\eta_{1}, \eta_{2}\right)_{\mathcal{M}}
$$

Then, as in $[12,14]$, we define

$$
\eta=\eta^{t}(x, s)=u(x, t)-u(x, t-s), \quad s \in \mathbb{R}^{+} .
$$

Using this new variable $\eta$ we can reformulate the system (1.1)-(1.3) to become

$$
\left\{\begin{array}{l}
u_{t t}+A u_{t t}+A u-\int_{0}^{\infty} \mu(s) A \eta(s) d s+f(u)=h, \\
\eta_{t}=T \eta+u_{t}
\end{array}\right.
$$

with initial conditions

$$
u(0)=u_{0}, \quad u_{t}(0)=v_{0}, \quad \eta^{0}(s)=\eta_{0},
$$

where $\eta_{0}(s)=u_{0}-\varphi(s)$ for all $s \in \mathbb{R}^{+}$.

The system (2.1)-(2.2) is a particular case of the system considered in [9]. There, the authors established the well-posedness for a class of problems with $\left|u_{t}\right|^{\rho} u_{t t}$ instead of $u_{t t}$ as in (2.1). They proved, among other results, that the system (2.1)-(2.2) with initial data $z=\left(u_{0}, v_{0}, \eta_{0}\right) \in \mathcal{H}$ admits a unique weak solution

$$
(u, \eta) \in W^{2, \infty}\left(0, \tau ; H_{1}\right) \times C([0, \tau] ; \mathcal{M}),
$$

satisfying the identity

$$
\left(u_{t t}, \phi\right)+\left(u_{t t}, \phi\right)_{1}+(u, \phi)_{1}+\int_{0}^{\infty} \mu(s)(\eta(s), \phi)_{1} d s+(f(u), \phi)=(h, \phi)
$$

for every $\phi \in H_{1}$ and for a.e. $t>0$. Here, $\eta$ is a mild solution to the non-homogeneous linear equation in the Hilbert space $\mathcal{M}$,

$$
\frac{d}{d t} \eta=T \eta+u_{t}
$$


where $\tau$ is a positive real number arbitrarily fixed. In addition, it was shown in Estimate (4.4) of [9],

$$
\left\|u_{t t}\right\|_{1} \leq C, \quad \text { a.e. } t \in[0, \tau]
$$

where $C>0$ depends only on the initial data.

Now, due to the continuous dependence on initial data, the weak solution $(u, \eta)$ of the system (2.1)-(2.2), with initial data $\left(u(0), u_{t}(0), \eta^{0}\right)=z$, can be rewritten in the form

$$
S(t) z=\left(u(t), u_{t}(t), \eta^{t}\right)
$$

generating a $C_{0}$-semigroup $S(t)$ on $\mathcal{H}$.

We end this section by recalling that a global attractor for a $C_{0}$-semigroup $S(t)$ on $\mathcal{H}$ is a compact subset $\mathbf{A} \subset \mathcal{H}$ which is strictly invariant, that is, $S(t) \mathbf{A}=\mathbf{A}, \forall t \geq 0$, and uniformly attracting, that is,

$$
\operatorname{dist}_{\mathcal{H}}(S(t) B, \mathbf{A})=\sup _{x \in S(t) B} \inf _{y \in \mathbf{A}}\|x-y\|_{\mathcal{H}} \rightarrow 0 \quad \text { as } t \rightarrow \infty
$$

for any bounded set $B \subset \mathcal{H}$.

\section{Global attractors}

In this section we establish our main result. The assumptions we make in this paper are as follows.

$\left(\mathrm{H}_{1}\right)$ Assume $\mu \in C^{1}\left(\mathbb{R}^{+}\right) \cap L^{1}\left(\mathbb{R}^{+}\right)$and satisfying the following conditions:

(i) $\mu(s) \geq 0$ for all $s \in \mathbb{R}^{+}$;

(ii) there exists a positive constant $k_{1}$ such that $\mu^{\prime}(s) \leq-k_{1} \mu(s)$ for all $s \in \mathbb{R}^{+}$.

$\left(\mathrm{H}_{2}\right)$ The nonlinearity $f \in C^{1}(\mathbb{R})$ and verifies the following conditions:

(i) $|f(r)-f(s)| \leq C\left(1+|r|^{p}+|s|^{p}\right)|r-s|$ for all $r, s \in \mathbb{R}$, where $0 \leq p<4$;

(ii) there exists a positive constant $\rho$ such that $f(s) s-\hat{f}(s) \geq-\rho$, where $\hat{f}(s)=\int_{0}^{s} f(\tau) d \tau$.

$\left(\mathrm{H}_{3}\right)$ The forcing $h$ belongs to the dual space of $H_{1}$.

To simplify the notation we write $\|\mu\|_{L^{1}\left(\mathbb{R}^{+}\right)}=k_{0}$ in our estimates. We also observe that the energy associated with the problem (2.1)-(2.2) is given by

$$
\begin{aligned}
E(t)= & \frac{1}{2}\left\|u_{t}\right\|^{2}+\frac{1}{2}\left\|u_{t}\right\|_{1}^{2}+\frac{1}{2}\|u\|_{1}^{2}+\frac{1}{2}\|\eta\|_{\mathcal{M}}^{2} \\
& +\int_{\Omega} \hat{f}(u) d x-\int_{\Omega} h u d x, \quad t \geq 0 .
\end{aligned}
$$

Our main result is the following.

Theorem 3.1 Suppose that the conditions $\left(\mathrm{H}_{1}\right)-\left(\mathrm{H}_{3}\right)$ are verified. Then the dynamical system $(S(t), \mathcal{H})$ generated by the problem (2.1)-(2.2) has a global attractor.

The proof of this theorem will be completed at the end of this section. 


\subsection{Abstract theory}

Let us present a small collection of well-known results of from the theory of attractors. This can be found in, e.g., [17-21]. A dynamical system $(\mathcal{H}, S(t))$ is called dissipative if the semigroup $S(t)$ has an absorbing set, that is, a bounded set $B_{0} \subset \mathcal{H}$ such that

$$
S(t) B \subset B_{0}, \quad \forall t \geq t_{B},
$$

for all bounded set $B \subset \mathcal{H}$. A semigroup $S(t)$ is asymptotically smooth in $\mathcal{H}$ if for any bounded positive invariant set $B \subset \mathcal{H}$, that is, $S(t) B \subseteq B$ for all $t \geq 0$, there exists a compact set $K \subset \bar{B}$, such that

$$
\operatorname{dist}_{\mathcal{H}}(S(t) B, K) \rightarrow 0 \quad \text { as } t \rightarrow \infty
$$

Then a classical result asserts that a dissipative $C_{0}$-semigroup $S(t)$ defined on $\mathcal{H}$ has a compact global attractor in $\mathcal{H}$ if and only if it is asymptotically smooth in $\mathcal{H}$.

Now, it is well known from Proposition 2.10 in [18] that a $S(t)$ is asymptotically smooth in $\mathcal{H}$ if for any positively invariant set $B \subset \mathcal{H}$, and for any $\varepsilon>0$, there exists $T=T(\varepsilon, B)$ such that

$$
\|S(T) x-S(T) y\|_{\mathcal{H}} \leq \varepsilon+\phi_{T}(x, y), \quad \forall x, y \in B,
$$

where $\phi_{T}: B \times B \rightarrow \mathbb{R}$ satisfies

$$
\liminf _{n \rightarrow \infty} \liminf _{m \rightarrow \infty} \phi_{T}\left(z_{n}, z_{m}\right)=0,
$$

for any sequence $\left(z_{n}\right)$ in $B$.

\subsection{Dissipativeness}

In this section we shall construct a bounded absorbing set to our system $(\mathcal{H}, S(t))$ where $S(t)$ is the solution operator defined in $(2.5)$. Let $\left(u, u_{t}, \eta\right)$ be a weak solution of the system (2.1)-(2.2). Since $H_{1}$ is dense in $H_{2}$ and $u \in L^{\infty}\left(0, \tau ; H_{1}\right)$ we can assume that $u$ is more regular and obtain

$$
\|u\|_{L^{p+2}}^{p+2} \leq K_{\Omega}\|u\|_{1}^{p+2}\|u\|
$$

where $K_{\Omega}$ is an embedding constant. To simplify the notation denote all the embedding constants by $C_{\Omega}$. Replacing $\phi$ by $u_{t}$ in (2.3) and adding the identity $\left(\eta_{t}, \eta\right)=(T \eta, \eta)_{\mathcal{M}}+$ $\left(u_{t}, \eta\right)_{\mathcal{M}}$ we get

$$
\frac{d}{d t} E(t)=(T \eta, \eta)_{\mathcal{M}}=\frac{1}{2} \int_{0}^{\infty} \mu^{\prime}(s)\|\eta(s)\|_{1}^{2} d s \leq 0 .
$$

We are going to apply the perturbed energy method. We consider the maps

$$
\Psi(t)=\left(u_{t}(t), u(t)\right)+\left(u_{t}(t), u(t)\right)_{1}, \quad \Phi(t)=\left(u_{t}(t), \eta^{t}\right)_{\mathcal{M}}+\left(u_{t}(t), \eta^{t}\right)_{\mathcal{M}_{0}}
$$


and

$$
F_{\varepsilon}(t)=\varepsilon^{-1} E(t)+\varepsilon \Psi(t)-\Phi(t)
$$

where $0<\varepsilon \leq 1$. Then we have the following result.

Lemma 3.2 Let $\Psi, \Phi$ and $F_{\varepsilon}$ be the maps defined in (3.3) and (3.4). Then

(a) there exist constants $\beta=\beta\left(k_{0}, \lambda_{1}\right) \geq 1$ and $C_{\rho, \lambda_{1}, h, \Omega} \geq 0$ such that

$$
|\varepsilon \Psi(t)-\Phi(t)| \leq \beta\left(E(t)+C_{\rho, \lambda, h, \Omega}\right)
$$

(b) for every $0<\varepsilon<\beta^{-1}$, the positive constants $\beta_{1}=\varepsilon^{-1}-\beta$ and $\beta_{2}=\varepsilon^{-1}+\beta$ satisfy the inequality

$$
\beta_{1} E(t)-\frac{1}{2} C_{\rho, \lambda_{1}, h, \Omega} \leq F_{\varepsilon}(t) \leq \beta_{2} E(t)+\frac{1}{2} C_{\rho, \lambda_{1}, h, \Omega}
$$

Proof By using Sobolev embeddings and condition $\left(\mathrm{H}_{2}\right)$ we have

$$
\begin{aligned}
\left|-\int_{\Omega}(\hat{f}(u)-h u) d x\right| & =\left|-\int_{\Omega}(\hat{f}(u)-f(u) u+f(u) u-h u) d x\right| \\
& \leq\left|\int_{\Omega}(\hat{f}(u)-f(u) u) d x\right|+\left|\int_{\Omega}(f(u) u-h u) d x\right| \\
& \leq \rho|\Omega|+\int_{\Omega}|(f(u) u-h u)| d x \\
& \leq \rho|\Omega|+\int_{\Omega}\left(1+|u|^{q}\right)|u|^{2} d x+6 \lambda_{1}^{-1}\|h\|^{2}+\frac{1}{12}\|u\|_{1}^{2} \\
& \leq \rho|\Omega|+\|u\|^{2}+\|u\|_{p+2}^{p+2}+6 \lambda_{1}^{-1}\|h\|^{2}+\frac{1}{12}\|u\|_{1}^{2} \\
\leq & \leq|\Omega|+K_{\Omega}\|u\|_{1}+K_{\Omega}\|u\|_{1}+6 \lambda_{1}^{-1}\|h\|^{2}+\frac{1}{12}\|u\|_{1}^{2} \\
\leq & \\
& +6|\Omega|+12\left(K_{\Omega}\right)^{2}+\frac{1}{12}\|u\|_{1}^{2}+\frac{1}{12}\|u\|_{1}^{2} \\
& +h\left\|^{2}+\frac{1}{12}\right\| u \|_{1}^{2},
\end{aligned}
$$

where $|\Omega|$ is the measure of $\Omega$. Therefore, there is a positive constant $C_{\rho, \lambda_{1}, h, \Omega}$ such that

$$
\frac{1}{4}\|u\|_{1}^{2}+\int_{\Omega}(\hat{f}(u)-h u) d x+C_{\rho, \lambda_{1}, h, \Omega} \geq 0
$$

Applying the inequality (3.6) we obtain

$$
\begin{aligned}
|\varepsilon \Psi(t)-\Phi(t)| \leq & \varepsilon\left|\left(u_{t}, u\right)+\left(u_{t}, u\right)_{1}\right|+\left|\left(u_{t}, \eta\right)_{\mathcal{M}}+\left(u_{t}, \eta\right)_{\mathcal{M}_{0}}\right| \\
\leq & \varepsilon\left(2 \lambda_{1}^{-1}\left\|u_{t}\right\|^{2}+\frac{1}{8}\|u\|_{1}^{2}\right)+\varepsilon\left(2\left\|u_{t}\right\|_{1}^{2}+\frac{1}{8}\|u\|_{1}^{2}\right) \\
& +\left(k_{0}\left\|u_{t}\right\|_{1}^{2}+\frac{1}{4}\|\eta\|_{\mathcal{M}}^{2}\right)+\left(k_{0}\left\|u_{t}\right\|^{2}+\frac{1}{4}\|\eta\|_{\mathcal{M}}^{2}\right)
\end{aligned}
$$




$$
\begin{aligned}
= & \left(k_{0}+2 \lambda_{1}^{-1} \varepsilon\right)\left\|u_{t}\right\|^{2}+\left(k_{0}+2 \varepsilon\right)\left\|u_{t}\right\|_{1}^{2}+\frac{\varepsilon}{4}\|u\|_{1}^{2}+\frac{1}{2}\|\eta\|_{\mathcal{M}}^{2} \\
\leq & \left(k_{0}+2 \lambda_{1}^{-1} \varepsilon\right)\left\|u_{t}\right\|^{2}+\left(k_{0}+2 \varepsilon\right)\left\|u_{t}\right\|_{1}^{2}+\frac{\varepsilon}{4}\|u\|_{1}^{2}+\frac{1}{2}\|\eta\|_{\mathcal{M}}^{2} \\
& +\left(\frac{1}{4}\|u\|_{1}^{2}+\int_{\Omega}(\hat{f}(u)-h u) d x+C_{\rho, \lambda_{1}, h, \Omega}\right) \\
\leq & \beta\left(E(t)+C_{\rho, \lambda_{1}, h, \Omega}\right),
\end{aligned}
$$

where $\beta=\sup _{0<\varepsilon \leq 1}\left\{1, \varepsilon, 2\left(k_{0}+2 \varepsilon\right), 2\left(k_{0}+2 \lambda_{1}^{-1} \varepsilon\right)\right\}$, which ends the proof of (a). Now, let $0<\varepsilon<\frac{1}{\beta}$. By item (a) we obtain

$$
\begin{aligned}
\left|F_{\varepsilon}(t)-\varepsilon^{-1} E(t)\right| & =|\varepsilon \Psi(t)-\Phi(t)| \\
& \leq \beta\left(E(t)+C_{\rho, \lambda_{1}, h, \Omega}\right),
\end{aligned}
$$

which provides

$$
\left(\frac{1}{\varepsilon}-\beta\right) E(t)-\frac{1}{2} C_{\rho, \lambda_{1}, h, \Omega} \leq F_{\varepsilon}(t) \leq\left(\frac{1}{\varepsilon}+\beta\right) E(t)+\frac{1}{2} C_{\rho, \lambda_{1}, h, \Omega} .
$$

This proves (b).

\section{Lemma 3.3 There exists $\varepsilon_{1}>0$ such that}

$$
F_{\varepsilon}^{\prime}(t) \leq-\varepsilon E(t)+C_{\varepsilon}, \quad \forall t \geq 0, \forall \varepsilon \in\left(0, \varepsilon_{1}\right) .
$$

Proof By (3.3) we get

$$
\begin{aligned}
\Psi^{\prime}(t)= & \left(u_{t t}, u\right)+\left(u_{t}, u_{t}\right)+\left(u_{t t}, u\right)_{1}+\left(u_{t}, u_{t}\right)_{1} \\
= & \left\|u_{t}\right\|^{2}+\left\|u_{t}\right\|_{1}^{2}-\|u\|_{1}^{2}-(u, \eta)_{\mathcal{M}}-(f(u), u)+(h, u) \\
\leq & \left\|u_{t}\right\|^{2}+\left\|u_{t}\right\|_{1}^{2}-\|u\|_{1}^{2}-(u, \eta)_{\mathcal{M}}+\rho|\Omega|+\left(-\int_{\Omega} \hat{f}(u) d x+(h, u)\right) \\
= & \left\|u_{t}\right\|^{2}+\left\|u_{t}\right\|_{1}^{2}-\|u\|_{1}^{2}-(u, \eta)_{\mathcal{M}}+\rho|\Omega|-E(t)+\frac{1}{2}\left\|u_{t}\right\|^{2}+\frac{1}{2}\left\|u_{t}\right\|_{1}^{2} \\
& +\frac{1}{2}\|u\|_{1}^{2}+\frac{1}{2}\|\eta\|_{\mathcal{M}}^{2} \\
\leq & -E(t)+\frac{3}{2}\left\|u_{t}\right\|^{2}+\frac{3}{2}\left\|u_{t}\right\|_{1}^{2}-\frac{1}{2}\|u\|_{1}^{2}+\frac{1}{2}\|\eta\|_{\mathcal{M}}^{2}-(u, \eta)_{\mathcal{M}}+\rho|\Omega| \\
\leq & -E(t)+\left(1+\lambda_{1}^{-1}\right)\left\|u_{t}\right\|_{1}^{2}-\frac{1}{2}\|u\|_{1}^{2}+\frac{1}{2}\|\eta\|_{\mathcal{M}}^{2}+\frac{1}{4}\|u\|_{1}^{2}+k_{0}\|\eta\|_{\mathcal{M}}^{2}+\rho|\Omega| \\
\leq & -E(t)+\left(1+\lambda_{1}^{-1}\right)\left\|u_{t}\right\|_{1}^{2}-\frac{1}{4}\|u\|_{1}^{2}+\left(\frac{1}{2}+k_{0}\right)\|\eta\|_{\mathcal{M}}^{2}+\rho|\Omega|
\end{aligned}
$$

and

$$
\begin{aligned}
-\Phi^{\prime}(t) & =-\left(u_{t t}, \eta\right)_{\mathcal{M}}-\left(u_{t}, \eta_{t}\right)_{\mathcal{M}}-\left(u_{t t}, \eta\right)_{\mathcal{M}_{0}}-\left(u_{t}, \eta_{t}\right)_{\mathcal{M}_{0}} \\
& =-\left(u_{t t}, \eta\right)_{\mathcal{M}}-\left(u_{t t}, \eta\right)_{\mathcal{M}_{0}}-\left\|u_{t}\right\|_{\mathcal{M}}^{2}-\left(T \eta, u_{t}\right)_{\mathcal{M}}-\left\|u_{t}\right\|_{\mathcal{M}_{0}}^{2}+\left(T \eta, u_{t}\right)_{\mathcal{M}_{0}} \\
& =-\left\|u_{t}\right\|_{\mathcal{M}}^{2}-\left\|u_{t}\right\|_{\mathcal{M}_{0}}^{2}-\left(T \eta, u_{t}\right)_{\mathcal{M}}-\left(T \eta, u_{t}\right)_{\mathcal{M}_{0}}+(u, \eta)_{\mathcal{M}}+(f(u), \eta)_{\mathcal{M}_{0}}
\end{aligned}
$$




$$
\begin{aligned}
& -(h, \eta)_{\mathcal{M}_{0}}-\int_{0}^{\infty} \int_{0}^{\infty} \mu(s) \mu(\tau)(\eta(\tau), \eta(s))_{1} d \tau d s \\
= & -\left\|u_{t}\right\|_{\mathcal{M}}^{2}-\left\|u_{t}\right\|_{\mathcal{M}_{0}}^{2}+\int_{0}^{\infty} \mu^{\prime}(s)\left(\eta, u_{t}\right)_{1} d s+\int_{0}^{\infty} \mu^{\prime}(s)\left(\eta, u_{t}\right) d s+(f(u), \eta)_{\mathcal{M}_{0}} \\
& -(h, \eta)_{\mathcal{M}_{0}}-\int_{0}^{\infty} \int_{0}^{\infty} \mu(s) \mu(\tau)(\eta(\tau), \eta(s))_{1} d \tau d s \\
\leq & \left.-\left\|u_{t}\right\|_{\mathcal{M}}^{2}-\left\|u_{t}\right\|_{\mathcal{M}_{0}}^{2}-k_{1}\left(\eta, u_{t}\right)\right)_{\mathcal{M}}-k_{1}\left(\eta, u_{t}\right)_{\mathcal{M}_{0}}+(f(u), \eta)_{\mathcal{M}_{0}}-(h, \eta)_{\mathcal{M}_{0}} \\
& -\int_{0}^{\infty} \int_{0}^{\infty} \mu(s) \mu(\tau)(\eta(\tau), \eta(s))_{1} d \tau d s \\
\leq & -\frac{1}{2}\left\|u_{t}\right\|_{\mathcal{M}}^{2}-\frac{1}{2}\left\|u_{t}\right\|_{\mathcal{M}_{0}}^{2}+k_{1}^{2}\|\eta\|_{\mathcal{M}}^{2}+k_{1}^{2}\|\eta\|_{\mathcal{M}_{0}}^{2}+(f(u), \eta)_{\mathcal{M}_{0}}-(h, \eta)_{\mathcal{M}_{0}} \\
& -\int_{0}^{\infty} \int_{0}^{\infty} \mu(s) \mu(\tau)(\eta(\tau), \eta(s))_{1} d \tau d s \\
\leq & -\frac{k_{0}}{2}\left(1+\lambda_{1}^{-1}\right)\left\|u_{t}\right\|_{1}+k_{1}^{2}\left(1+\lambda_{1}^{-1}\right)\|\eta\|_{\mathcal{M}}^{2}+\frac{\delta}{4}\|u\|_{1}^{2}+C_{\delta}\|\eta\|_{\mathcal{M}}^{2} \\
& +\frac{\lambda_{1}^{-1} k_{0}}{2}\|h\|^{2}+\frac{1}{2}\|\eta\|_{\mathcal{M}}^{2},
\end{aligned}
$$

where $0<\delta \leq 1$ and $C_{\delta}$ is a positive constant that verifies the inequality

$$
\begin{aligned}
\left|(f(u), \eta)_{\mathcal{M}_{0}}\right| & \leq \int_{0}^{\infty} \mu(s) \int_{\Omega}\left(1+|u|^{p}\right)|u||\eta| d x d s \\
& \leq \int_{0}^{\infty} \mu(s) \int_{\Omega}|u \| \eta| d x d s+\int_{0}^{\infty} \mu(s) \int_{\Omega}|u|^{p+1}|\eta| d x d s \\
& \leq \lambda_{1}^{-1} \int_{0}^{\infty} \mu(s)\|u\|_{1}\|\eta\|_{1} d s+\int_{0}^{\infty} \mu(s)\|u\|_{p+2}^{p+1}\|\eta\|_{p+2} d s \\
& \leq \lambda_{1}^{-1} \int_{0}^{\infty} \mu(s)\|u\|_{1}\|\eta\|_{1} d s+K_{\Omega} \int_{0}^{\infty} \mu(s)\|u\|_{1}\left\|_{\eta}\right\|_{1} d s \\
& \leq K_{\Omega}\|u\|_{1} \int_{0}^{\infty} \mu(s)\|\eta\|_{1} d s \\
& \leq \frac{\delta}{4}\|u\|_{1}^{2}+C_{\delta}\|\eta\|_{\mathcal{M}}^{2} .
\end{aligned}
$$

Now, for every $0<\varepsilon<\frac{k_{0}}{2}$, the above inequalities provide

$$
\begin{aligned}
F_{\varepsilon}^{\prime}(t)-\varepsilon^{-1} E^{\prime}(t) & =\varepsilon \Psi^{\prime}(t)-\Phi^{\prime}(t) \\
& \leq\left(1+\lambda_{1}^{-1}\right)\left(\varepsilon-\frac{k_{0}}{2}\right)\left\|u_{t}\right\|_{1}-\varepsilon E(t)+\frac{1}{4}(\delta-\varepsilon)\|u\|_{1}^{2}+\tilde{C}_{\varepsilon, \delta} \\
& \leq-\varepsilon E(t)+\frac{1}{4}(\delta-\varepsilon)\|u\|_{1}^{2}+\tilde{C}_{\varepsilon, \delta}
\end{aligned}
$$

where $\tilde{C}_{\varepsilon, \delta}$ is a positive constant. As $E^{\prime}(t) \leq 0$ we can choose $\delta \leq \varepsilon$ in the previous inequality to obtain

$$
F_{\varepsilon}^{\prime}(t) \leq-\varepsilon E(t)+C_{\varepsilon}
$$

which ends the proof of the lemma. 
Lemma 3.4 (Absorbing set) Let $S(t)$ be the $C_{0}$-semigroup defined in (2.5). Then $(\mathcal{H}, S(t))$ is a dissipative dynamical system.

Proof We shall prove that $S(t)$ has a bounded absorbing set. Let $\varepsilon_{0}=\min \left\{\frac{1}{2 \beta}, \varepsilon_{1}\right\}$. By item (b) of Lemma 3.2 we have

$$
\beta_{1} E(t)-\frac{1}{2} C_{\rho, \lambda_{1}, h, \Omega} \leq F_{\varepsilon}(t) \leq \beta_{2} E(t)+\frac{1}{2} C_{\rho, \lambda_{1}, h, \Omega},
$$

where $\beta_{1}=\varepsilon^{-1}-\beta$ and $\beta_{2}=\varepsilon^{-1}+\beta$. Multiplying the inequality (3.7) for $\frac{\varepsilon}{\beta_{2}}$ we get

$$
\frac{\varepsilon}{\beta_{2}} F_{\varepsilon}(t) \leq \varepsilon E(t)+\mathbf{a}_{\varepsilon}
$$

where $\mathbf{a}_{\varepsilon}=\varepsilon \beta_{2}^{-1} C_{\rho, \lambda_{1}, h, \Omega}$. Now by Lemma 3.3 we have

$$
F_{\varepsilon}^{\prime}(t) \leq-\varepsilon E(t)+C_{\varepsilon} .
$$

Adding the inequalities (3.8) and (3.9) we obtain

$$
F_{\varepsilon}^{\prime}(t)+\frac{\varepsilon}{\beta_{2}} F_{\varepsilon}(t) \leq \mathbf{b}_{\varepsilon}
$$

where $\mathbf{b}_{\varepsilon}=\mathbf{a}_{\varepsilon}+C_{\varepsilon}$. By (3.10) we conclude that

$$
F_{\varepsilon}(t) \leq\left(F_{\varepsilon}(0)-\varepsilon^{-1} \mathbf{b}_{\varepsilon} \beta_{2}\right) e^{-\frac{\varepsilon}{\beta_{2}} t}+\varepsilon^{-1} \mathbf{b}_{\varepsilon} \beta_{2}, \quad \text { for all } t \geq 0 .
$$

But by inequality (3.8) we have $F_{\varepsilon}(0) \leq \beta_{2} E(0)+\varepsilon^{-1} \mathbf{a}_{\varepsilon} \beta_{2}$. Therefore, by inequality (3.11) we get

$$
F_{\varepsilon}(t) \leq\left(\beta_{2} E(0)-\varepsilon^{-1} C_{\varepsilon} \beta_{2}\right) e^{-\frac{\varepsilon}{\beta_{2}} t}+\varepsilon^{-1} \mathbf{b}_{\varepsilon} \beta_{2}, \quad \text { for all } t \geq 0 .
$$

Combining (3.7) and (3.12) we obtain

$$
\begin{aligned}
E(t) & \leq \frac{\beta_{2}}{\beta_{1}}\left(E(0)-\varepsilon^{-1} C_{\varepsilon}\right) e^{-\frac{\varepsilon}{\beta_{2}} t}+\frac{\beta_{2}}{\beta_{1}} C_{\varepsilon}+\frac{3}{2 \beta_{1}} C_{\rho, \lambda_{1}, h, \Omega} \\
& \leq \frac{\beta_{2}}{\beta_{1}} E(0) e^{-\frac{\varepsilon}{\beta_{2}} t}+\frac{\beta_{2}}{\beta_{1}} C_{\varepsilon}+\frac{3}{2 \beta_{1}} C_{\rho, \lambda_{1}, h, \Omega} .
\end{aligned}
$$

By (3.6) we have

$$
E(t) \geq \sigma\left\|\left(u(t), u_{t}(t), \eta^{t}\right)\right\|_{\mathcal{H}}^{2}-C_{\rho, \lambda_{1}, h, \Omega}, \quad \text { for all } t \geq 0,
$$

where $\sigma=\min \left\{1, \frac{\lambda_{1}}{2}\right\}$. Combining (3.13) and (3.14) we get

$$
\left\|\left(u(t), u_{t}(t), \eta^{t}\right)\right\|_{\mathcal{H}}^{2} \leq \frac{\beta_{2}}{\beta_{1} \sigma} E(0) e^{-\frac{\varepsilon}{\beta_{2}} t}+\frac{\beta_{2}}{\beta_{1} \sigma} C_{\varepsilon}+\left(\frac{3}{2 \beta_{1} \sigma}+\frac{1}{\sigma}\right) C_{\rho, \lambda_{1}, h, \Omega} .
$$

By inequality (3.15) the ball $B(0, R) \subset \mathcal{H}$, where

$$
R>\sqrt{\frac{2 \beta_{2}}{\beta_{1} \sigma} C_{\varepsilon}+\left(\frac{3}{\beta_{1} \sigma}+\frac{2}{\sigma}\right) C_{\rho, \lambda_{1}, h, \Omega}},
$$

is an absorbing set of the semigroup $S(t)$. 


\subsection{Compactness}

In this section we shall prove that the system $(\mathcal{H}, S(t))$ is asymptotically smooth.

Lemma 3.5 (Stabilization inequality) Let $B \subset \mathcal{H}$ be a bounded invariant set and $z=$ $\left(u_{0}, v_{0}, \eta_{0}\right), \tilde{z}=\left(\tilde{u}_{0}, \tilde{v}_{0}, \tilde{\eta}_{0}\right)$ two initial data in $B$. Then there exists $v>0$ such that

$$
\|S(t) z-S(t) \tilde{z}\|_{\mathcal{H}}^{2} \leq C_{B} e^{-v t}+C_{B} \int_{0}^{t}\left(\|w(s)\|_{L^{p+2}}^{2}+\left\|w_{t}(s)\right\|_{L^{p+2}}^{2}\right) d s
$$

where $(u, \eta),(\tilde{u}, \tilde{\eta})$ are the corresponding weak solutions of (2.1)-(2.2), $w=u-\tilde{u}$, and $C_{B}$ is a positive constant depending on $B$ but not on $t$.

Proof Let us also write $\xi=\eta-\tilde{\eta}$. Then $w$ is a weak solution of the system

$$
\left\{\begin{array}{l}
w_{t t}-\Delta w_{t t}-\Delta w-\int_{0}^{\infty} \mu(s) \Delta \xi(s) d s=f(u)-f(\tilde{u}), \\
\xi_{t}=T \xi+w_{t}
\end{array}\right.
$$

with Dirichlet boundary condition and initial data

$$
w(0)=u_{0}-\tilde{u}_{0}, \quad w_{t}(0)=v_{0}-\tilde{v}_{0}, \quad \xi^{0}=\eta_{0}=\tilde{\eta}_{0} .
$$

We define the energy functional

$$
G(t)=\frac{1}{2}\left\|w_{t}(t)\right\|^{2}+\frac{1}{2}\left\|w_{t}(t)\right\|_{1}^{2}+\frac{1}{2}\|w(t)\|_{1}^{2}+\frac{1}{2}\left\|\xi^{t}\right\|_{\mathcal{M}}^{2} .
$$

In the following, $C_{0}$ will denote several positive constants dependent on $B$ but not on $t$.

Claim 1 There exists a constant $C_{0}>0$ such that

$$
G^{\prime}(t) \leq \frac{1}{2} \int_{0}^{\infty} \mu^{\prime}(s)\left\|\xi^{t}(s)\right\|_{1}^{2} d s+C_{0}\left(\|w(t)\|_{L^{p+2}}^{2}+\left\|w_{t}(t)\right\|_{L^{p+2}}^{2}\right) .
$$

To prove the claim, we multiply the first equation in (3.17) by $w_{t}$ and integrate over $\Omega$. Then we obtain

$$
G^{\prime}(t)=\frac{1}{2} \int_{0}^{\infty} \mu^{\prime}(s)\|\xi(s)\|_{1}^{2} d s-\int_{\Omega}(f(u)-f(\tilde{u})) w_{t} d x .
$$

Using $\left(\mathrm{H}_{2}\right)$ we have

$$
\begin{aligned}
\left|\int_{\Omega}(f(u)-f(\tilde{u})) w_{t} d x\right| & \leq C_{f}\left(1+\|u\|_{L^{p+2}}^{p}+\|\tilde{u}\|_{L^{p+2}}^{p}\right)\|w\|_{L^{p+2}}\left\|w_{t}\right\|_{L^{p+2}} \\
& \leq C_{0}\left(\|w(t)\|_{L^{p+2}}^{2}+\left\|w_{t}(t)\right\|_{L^{p+2}}^{2}\right)
\end{aligned}
$$

since $B$ is bounded and invariant. Then we see that (3.18) holds.

Now, let us define the perturbed functional

$$
J(t)=N G(t)+\varepsilon \tilde{\Psi}(t)+\tilde{\Phi}(t),
$$


where

$$
\tilde{\Psi}(t)=\left(w(t), w_{t}(t)\right)+\left(w(t), w_{t}(t)\right)_{1}, \quad \tilde{\Phi}(t)=\left(w(t), \xi^{t}\right)_{\mathcal{M}}+\left(w(t), \xi^{t}\right)_{\mathcal{M}_{0}},
$$

and $N \geq 1,0<\varepsilon<1$ are constants to be determined. Then the following claims can be proved with similar arguments to the above one and to the proof of the absorbing set.

Claim 2 There exist constants $\beta_{1}, \beta_{2}, C_{\beta}>0$ such that, if $N>C_{\beta}$,

$$
\beta_{1} G(t) \leq J(t) \leq \beta_{2} G(t), \quad t \geq 0 .
$$

Claim 3 There exists a constant $C_{1}>0$ such that

$$
\begin{aligned}
\tilde{\Psi}^{\prime}(t) \leq & -G(t)-\frac{1}{4}\|w(t)\|_{1}^{2}+\frac{3}{2}\left\|w_{t}(t)\right\|^{2}+\frac{3}{2}\left\|w_{t}(t)\right\|_{1}^{2} \\
& -C_{1} \int_{0}^{\infty} \mu^{\prime}(s)\left\|\xi^{t}(s)\right\|_{1}^{2} d s+C_{0}\|w(t)\|_{L^{p+2}}^{2} .
\end{aligned}
$$

Claim 4 Given $\delta>0$ there exists a constant $C_{\delta}>0$ such that

$$
\tilde{\Phi}^{\prime}(t) \leq \delta\|w(t)\|_{1}^{2}-\frac{k_{0}}{2}\left\|w_{t}(t)\right\|_{1}^{2}-C_{\delta} \int_{0}^{t} \mu^{\prime}(s)\left\|\xi^{t}(s)\right\|_{1}^{2} d s
$$

Now, taking $\varepsilon>0$ sufficiently small and $N>0$ sufficiently large, we obtain from (3.18), (3.20), and (3.21),

$$
J^{\prime}(t) \leq-\varepsilon G(t)+C_{0}\left(\|w(t)\|_{L^{p+2}}^{2}+\left\|w_{t}(t)\right\|_{L^{p+2}}^{2}\right), \quad t \geq 0 .
$$

Combining this with (3.19) we have, as in the proof of the absorbing set,

$$
G(t) \leq \frac{\beta_{2}}{\beta_{1}} G(0) e^{-\frac{\varepsilon}{\beta_{2}} t}+C_{0} \int_{0}^{t} e^{-\frac{\varepsilon}{\beta_{2}}(t-s)}\left(\|w(s)\|_{L^{p+2}}^{2}+\left\|w_{t}(s)\right\|_{L^{p+2}}^{2}\right) d s, \quad \forall t \geq 0 .
$$

This implies (3.16) by taking $v=\varepsilon / \beta_{2}$ and in view of the definition of $G(t)$.

Lemma 3.6 (Asymptotic smoothness) Let $S(t)$ be the $C_{0}$-semigroup defined in (2.5). Then the system $(\mathcal{H}, S(t))$ is asymptotically smooth.

Proof We apply the compactness criterion presented in Proposition 2.10 of [18]. As recalled in Section 3.1, we must check conditions (3.1) and (3.2).

Given a forward invariant set $B \subset \mathcal{H}$ and $\varepsilon>0$, we can take $T>0$ such that

$$
\sqrt{2 C_{B}} e^{-\frac{v}{2} T}<\varepsilon
$$

Then from (3.16), using notation

$$
S(t) z^{n}=\left(u^{n}(t), u_{t}^{n}(t), \eta_{n}^{t}\right),
$$


we obtain for any $z^{1}, z^{2} \in B$,

$$
\left\|S(t) z^{1}-S(t) z^{2}\right\|_{\mathcal{H}} \leq \varepsilon+\left(2 C_{B} \int_{0}^{T}\left\|u^{1}-u^{2}\right\|_{L^{p+2}}^{2}+\left\|u_{t}^{1}-u_{t}^{2}\right\|_{L^{p+2}}^{2} d s\right)^{\frac{1}{2}}
$$

with $0<t<T$. Then defining

$$
\phi_{T}\left(z^{1}, z^{2}\right)=\sqrt{2 C_{B}}\left(\int_{0}^{T}\left\|u^{1}(s)-u^{2}(s)\right\|_{L^{p+2}}^{2}+\left\|u_{t}^{1}(s)-u_{t}^{2}(s)\right\|_{L^{p+2}}^{2} d s\right)^{\frac{1}{2}},
$$

we see that condition (3.1) holds.

It remains to show that (3.2) also holds. Given any sequence $\left(z^{n}\right) \subset B$, from the positive invariance of $B$ we see that $S(t) z^{n}=\left(u^{n}(t), u_{t}^{n}(t), \eta_{n}^{t}\right)$ is uniformly bounded in $\mathcal{H}$. Then we conclude that

$$
\begin{aligned}
& u^{n} \quad \text { is bounded in } L^{\infty}\left(0, T, H_{1}\right), \\
& u_{t}^{n} \quad \text { is bounded in } L^{\infty}\left(0, T, L^{2}(\Omega)\right) \cap L^{\infty}\left(0, T, H_{1}\right),
\end{aligned}
$$

and from (2.4),

$$
u_{t t}^{n} \quad \text { is bounded in } L^{2}\left(0, T, L^{2}(\Omega)\right) \text {. }
$$

Then from Simon's theorem [22] we have

$$
u^{n}, u_{t}^{n} \quad \text { converge strongly in } C\left([0, T], L^{p+2}(\Omega)\right),
$$

since $H_{1}$ is compactly embedded in $L^{p+2}(\Omega)$. Therefore there is a subsequence such that

$$
\lim _{k \rightarrow \infty} \lim _{l \rightarrow \infty} \int_{0}^{T}\left\|u^{n_{k}}(s)-u^{n_{l}}(s)\right\|_{L^{p+2}}^{2}+\left\|u_{t}^{n_{k}}(s)-u_{t}^{n_{l}}(s)\right\|_{L^{p+2}}^{2} d s=0 .
$$

This shows that (3.2) also holds.

Proof of Theorem 3.1 Since we have proved that $(\mathcal{H}, S(t))$ is dissipative and asymptotically smooth, the existence of a global attractor follows from a classical result, as noticed in Section 3.1.

\section{Competing interests}

The authors declare that they have no competing interests.

\section{Authors' contributions}

All authors contributed equally to the manuscript and read and approved the final manuscript.

\section{Author details}

${ }^{1}$ College of Economic Mathematics, Southwestern University of Finance and Economics, Chengdu, 611130, P.R. China. ²Department of Sciences, Regional Campus of Goioerê, State University of Maringá, Goioerê, Paraná 87360-000, Brazil.

${ }^{3}$ Department of Mathematics, State University of Maringá, Maringá, Paraná 87020-900, Brazil.

\section{Acknowledgements}

This work was supported by the National Natural Science Foundation of China with grant number 11526164.

Received: 8 January 2016 Accepted: 31 January 2016 Published online: 08 February 2016 


\section{References}

1. Love, AEH: Treatise on Mathematical Theory of Elasticity. Dover, New York (1944)

2. Cavalcanti, MM, Domingos Cavalcanti, VN, Ferreira, J: Existence and uniform decay for a nonlinear viscoelastic equation with strong damping. Math. Methods Appl. Sci. 24, 1043-1053 (2001)

3. Han, X, Wang, M: Global existence and uniform decay for a nonlinear viscoelastic equation with damping. Nonlinear Anal. 70, 3090-3098 (2009)

4. Messaoudi, SA, Tatar, N-E: Exponential decay for a quasilinear viscoelastic equation. Math. Nachr. 282, 1443-1450 (2009)

5. Wu, S-T: General decay of solutions for a nonlinear system of viscoelastic wave equations with degenerate damping and source terms. J. Math. Anal. Appl. 406, 34-48 (2013)

6. Araújo, RO, Ma, TF, Qin, Y: Long-time behavior of a quasilinear viscoelastic equation with past history. J. Differ. Equ. 254, 4066-4087 (2013)

7. Qin, Y, Feng, B, Zhang, M: Uniform attractors for a non-autonomous viscoelastic equation with a past history. Nonlinear Anal. 101, 1-15 (2014)

8. Chen, C, Wang, H, Zhu, S: Global attractor and decay estimates of solutions to a class of nonlinear evolution equations. Math. Methods Appl. Sci. 34, 497-508 (2011)

9. Conti, M, Marchini, EM, Patta, V: A well posedness result for nonlinear viscoelastic equations with memory. Nonlinear Anal. 94, 206-216 (2014)

10. Cavalcanti, MM, Fatori, LH, Ma, TF: Attractors for wave equations with degenerate memory. J. Differ. Equ. 260, 56-83 (2016)

11. Gatti, S, Miranville, A, Pata, V, Zelik, S: Attractors for semi-linear equations of viscoelasticity with very low dissipation. Rocky Mt. J. Math. 38(4), 1117-1138 (2008)

12. Giorgi, C, Rivera, JE, Pata, V: Global attractors for a semilinear hyperbolic equation in viscoelasticity. J. Math. Anal. Appl. 260, 83-99 (2001)

13. Messaoudi, SA: General decay of solutions of a viscoelastic equation. J. Math. Anal. Appl. 341, 1457-1467 (2008)

14. Pata, V, Zucchi, A: Attractors for a damped hyperbolic equation with linear memory. Adv. Math. Sci. Appl. 11, 505-529 (2001)

15. Lions, JL: Quelques Méthodes de Résolution des Problèmes aux Limites non Linéaires. Dunod, Paris (1969)

16. Dafermos, CM: Asymptotic stability in viscoelasticity. Arch. Ration. Mech. Anal. 37, 297-308 (1970)

17. Babin, AV, Vishik, MI: Attractors of Evolution Equations. Studies in Mathematics and Its Applications, vol. 25. North-Holland, Amsterdam (1992)

18. Chueshov, I, Lasiecka, I: Long-time behavior of second order evolution equations with nonlinear damping. Mem. Am. Math. Soc. 195, 912 (2008)

19. Hale, JK: Asymptotic Behaviour of Dissipative Systems. Mathematical Surveys and Monographs, vol. 25. Am. Math. Soc., Providence (1988)

20. Ladyzhenskaya, O: Attractors for Semi-Groups and Evolution Equations. Cambridge University Press, Cambridge (1991)

21. Temam, R: Infinite Dimensional Dynamical Systems in Mechanics and Physics. Springer, New York (1997)

22. Simon, J: Compact sets in the space $L^{P}(0, T ; B)$. Ann. Mat. Pura Appl. 146, 65-96 (1987)

\section{Submit your manuscript to a SpringerOpen ${ }^{\circ}$ journal and benefit from:}

- Convenient online submission

- Rigorous peer review

- Immediate publication on acceptance

- Open access: articles freely available online

- High visibility within the field

- Retaining the copyright to your article 